\title{
Für die Kinder nur das Natürlichste
}

Wenn es um den Nachwuchs geht, setzen immer mehr Eltern auf die Komplementärmedizin. Sie versuchen ohne Medikamente auszukommen, so lange es geht. Jetzt ist auch die Forschung zum Thema «Kinder und Ganzheitsmedizin» in Schwung gekommen.

Viele Eltern misstrauen der Schulmedizin, wenn es um die Behandlung von Kinderkrankheiten geht, und greifen häufig zu Hausmitteln und Methoden der Ganzheitsmedizin. Während einige wenige Eltern Extrempositionen einnehmen und etwa das Impfen kategorisch ablehnen, gehen die meisten sehr pragmatisch mit der Frage um, wann sie Methoden der Komplementärmedizin und wann sie solche der Schulmedizin vom Arzt verlangen sollen: Wenn es sich um leichte Erkrankungen handelt, setzen sie häufig auf die «sanften» Methoden.

Mittlerweile hat sich auch bei den meisten Haus- und Kinderärzten in der Schweiz die Haltung herauskristallisiert, dass Bagatellerkrankungen ohne Weiteres mit den Methoden der Komplementärmedizin behandelt werden können, wohingegen ernstere und besonders lebensbedrohliche Erkrankungen zwingend mit schulmedizinischen Methoden in den Griff bekommen werden sollen.

Während es aus der Schweiz und dem restlichen deutschsprachigen Raum nur wenige Angaben über die Häufigkeit der komplementärmedizinischen Behandlung für Kinder und Jugendliche gibt, liegen mittlerweile aus den USA umfangreiche Untersuchungen vor, die sich mit der Frage beschäftigen, wie gut die Komplementärmedizin in der Pädiatrie heute verankert ist. Nach einer vor knapp einem Jahr veröffentlichten Untersuchung (Birdee GS, et al. Pediatrics 2010;125:249-256) benutzen jedes Jahr etwa 8,7 Millionen amerikanische Kinder und Jugendliche die Methoden der Komplementär- und Ganzheits- medizin; das entspricht der Anwendung bei $12 \%$ der Kinder in den USA.

\section{Bei Jugendlichen öfters angewendet}

Der Untersuchung liegen Daten von über 9000 Kindern zugrunde. Eines der interessantesten Ergebnisse: Die komplementärmedizinischen Verfahren werden häufiger bei Jugendlichen als bei Kindern unter 5 Jahren angewendet. Das deutet darauf hin, dass unerfahrene Eltern, die noch jüngere Kinder haben, eher auf Nummer sicher gehen wollen als solche, die schon Erfahrungen mit den Krankheiten des Nachwuchses haben.

Entscheidend für die Frage, ob die Kinder und Jugendlichen mit komplementärmedizinischen Methoden behandelt werden, ist auch die Einstellung der Eltern. In der Studie wurde ein deutlicher Zusammenhang zwischen der Verwendung dieser Methoden bei den Eltern selbst und dem Einsatz bei den Kindern gefunden. Je höher ihre Bildung war, desto höher auch die Wahrscheinlichkeit, dass sie bei den Kinderärzten nach alternativen Methoden verlangten. Das Einkommen hatte nur auf die Auswahl der Verfahren einen Einfluss. In Haushalten, die mehr Geld zur Verfügung hatten, wurden bestimmte körperzentrierte Verfahren bei den Kindern häufiger angewendet.

Die Methoden der Komplementärmedizin, die bei den Kindern eingesetzt wurden, umfassten das ganze Spektrum und rangierten von der Akupunktur über Biofeedback bis zu Homöopathie und Chiropraxis. Am häufigsten wurden sie angewendet, um Rücken- oder Nackenschmerzen oder Halsweh, Entzündungen und Kopfschmerzen zu therapieren. Verbreitet wurden diese Verfahren auch eingesetzt, um z.B. Angst oder Stress, dermatologische Probleme, Schlaflosigkeit oder Übelkeit zu behandeln. Besonders häufig kamen sie zum Einsatz, um chronische Symptome zu lindern. Dazu gehörten auch Asthma, Nahrungsmittelallergien und die juvenile Arthritis.

\section{Wissenschaftliche Forschung}

Schon vor über 10 Jahren hat die «Amerikanische Akademie für Pädiatrie» die zunehmende Bedeutung der Komplementärmedizin für die Kinderheilkunde erkannt (Kemper KJ, et al. Pediatrics 2008;122:1374-1386). Die Nachfrage nach Behandlungsmethoden jenseits der Schulmedizin für Kinder ist seit Jahren angestiegen. Was allerdings mit dem Boom nicht standhalten konnte, war die wissenschaftliche Erforschung und Bewertung dieser Methoden in der Pädiatrie. Als eine der ersten Ärztegesellschaften hat sich die Amerikanische Akademie intensiv darum gekümmert, das vorhandene Wissen über die Wirkung der ganzheitlichen Behandlungsmethoden zu sichten, zu bewerten und den Praktikern zugänglich zu machen. In einer eigens gegründeten «Task Force» wurden Informationen gesammelt, die den State-of-the-Art in diesem Feld dokumentieren sollen. In regelmässigen Publikationen berichtet sie von ihren Ergebnissen, um die Kinderärzte bei der Anwendung dieser Methoden zu unterstützen. ( $r f i)$

\section{KARGER}

Fax +49 7614520714 Information@Karger.d www.karger.com 